\title{
Leiomiosarcoma intratesticular primario diagnosticado por biopsia hepática percutánea ecodirigida
}

\section{Intratesticular Leiomyosarcoma Diagnosed by Percutaneous Liver Biopsy}

\author{
M. Castellanos González ${ }^{1}$ \\ C. Delgado Martínez ${ }^{1}$ \\ M. Gómez Rubio ${ }^{1}$ \\ M. Butrón Vila ${ }^{2}$ \\ J. I. Arance $\mathrm{Gil}^{3}$ \\ B. de Cuenca Morón ${ }^{1}$
}
${ }^{1}$ Servicio del Aparato Digestivo del Hospital Universitario de Getafe, Getafe, Madrid, España
2 Servicio de Anatomía Patológica del Hospital Universitario de Getafe, Getafe, Madrid, España
${ }^{3}$ Servicio de Urología del Hospital Universitario de Getafe, Getafe, Madrid, España

\begin{abstract}
Address for correspondence M. Castellanos González, MD, Hospital Universitario de Getafe, Carretera, Toledo, Km 12,500, 28905 Getafe, Madrid, España (e-mail: marina_castellanos@hotmail.com).
\end{abstract}

Urol Colomb 2019;28:15-18.

\section{Resumen \\ Palabras Clave \\ - testículo \\ - leiomiosarcoma \\ - tumor intratesticular \\ - metástasis hepática \\ - punción biopsia hepática percutánea}

Se presenta el caso clínico de un varón de 80 años diagnosticado de leiomiosarcoma testicular primario, a raíz de alteraciones del perfil hepático en analítica sanguínea y lesiones ocupantes de espacio (LOE) hepatoesplénicas en ecografía abdominal. En primer lugar, se realizó punción de lesión hepática y posteriormente orquiectomía radical, revelando concordancia histológica, compatible con leiomiosarcoma intratesticular. Presentamos el caso clínico debido a la rareza de ese tipo de tumores, sobre todo en estadio III, y su peculiar diagnóstico, tras la detección de alteración hepática tanto analítica como ecográfica.

We present a case of an 80-year-old male diagnosed with primary testicular leiomyosarcoma due to the detection of hepatic profile analytical alterations and hepatosplenic space-occupying lesions in abdominal ultrasound. Puncture of hepatic lesion was performed first and radical orchiectomy later, revealing histological concordance, compatible with intratesticular leiomyosarcoma. We present the case due to the uncommon of this type of tumors, especially in stage III and its peculiar diagnosis, secondary of hepatic alteration, both analytic and ultrasound.

\section{Introducción}

El leiomiosarcoma es una neoplasia maligna de origen mesenquimal, localizada en tejidos con músculo liso. ${ }^{1}$

El leiomiosarcoma primario intratesticular es extremadamente raro, con apenas veinte casos descritos en la bibliografía. Todos ellos fueron diagnosticados en estadio I, localizados en el testículo, salvo uno que debutó con múltiples metátasis pulmonares, adenopatías paraaórticas y en otros órganos, compatible con estadio III. ${ }^{2-7}$

En este artículo, se recoge el caso de un paciente diagnosticado de leiomiosacarcoma intratesticular diseminado con metástasis hepáticas, esplénicas y pulmonares, a partir de biopsia hepática y pieza quirúrgica testicular.

\section{received}

July 1, 2018

accepted

August 14, 2018

published online

October 1, 2018
DOI https://doi.org/

10.1055/s-0038-1673337.

ISSN 0120-789X.

eISSN 2027-0119.
Copyright ( 2019 , Sociedad Colombiana License terms de Urología. Publicado por Thieme Revinter Publicações Ltda., Rio de Janeiro, Brazil. Todos los derechos reservados.

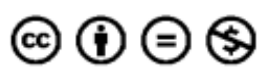




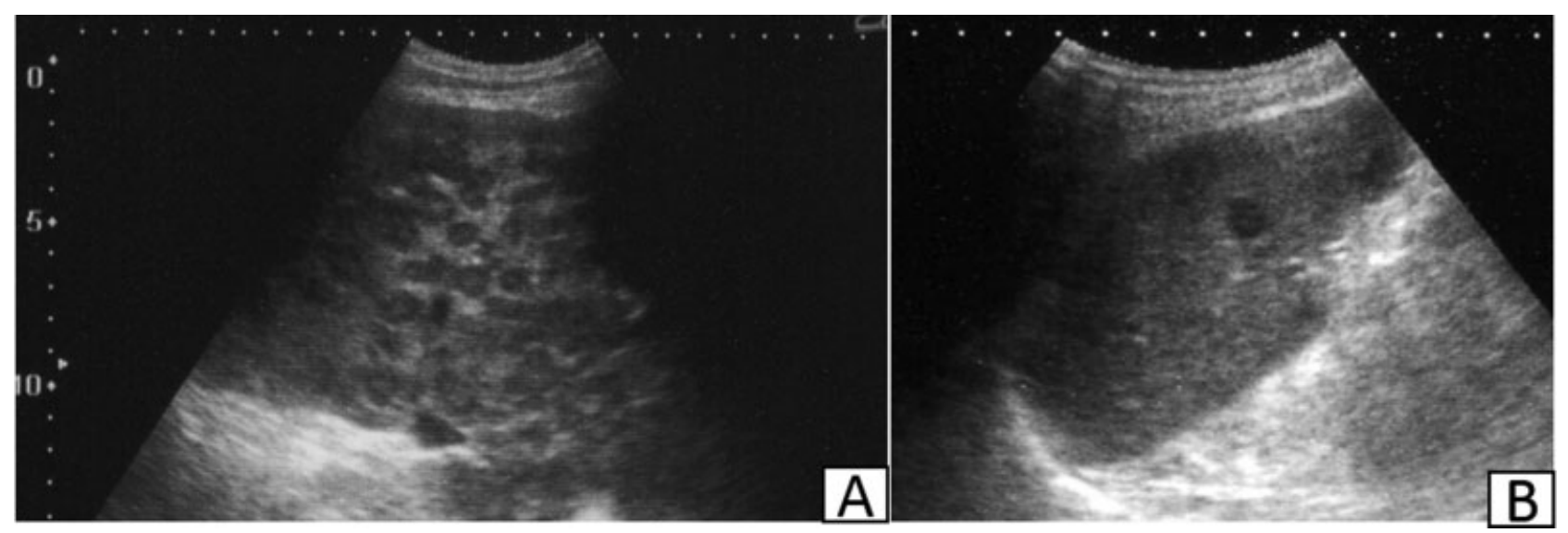

Fig. 1 Ecografía abdominal: Lesiones hepatoesplénicas ocupantes de espacio con patrón en “ojo de buey.” A. Higado. B. Bazo.

\section{Caso Clínico}

Varón de 80 años fumador, con antecedentes destacados de hipertrofia prostática benigna tratada con resección transuretral hace 14 años sin revisiones posteriores, hernioplastia inguinal bilateral y hemorroides. Consulta por síndrome constitucional, presentando en la analítica sanguínea alteraciones de perfil hepático y marcadores tumorales B2microgolubina y Ca 19.9 ligeramente elevados. Se realiza ecografía abdominal objetivando múltiples LOE hepatoesplénicas sólidas, sugerentes de metástasis (-Fig. 1). En revisión urológica se identifica teste izquierdo desestructurado y heterogéneo de gran tamaño (-Fig. 2).

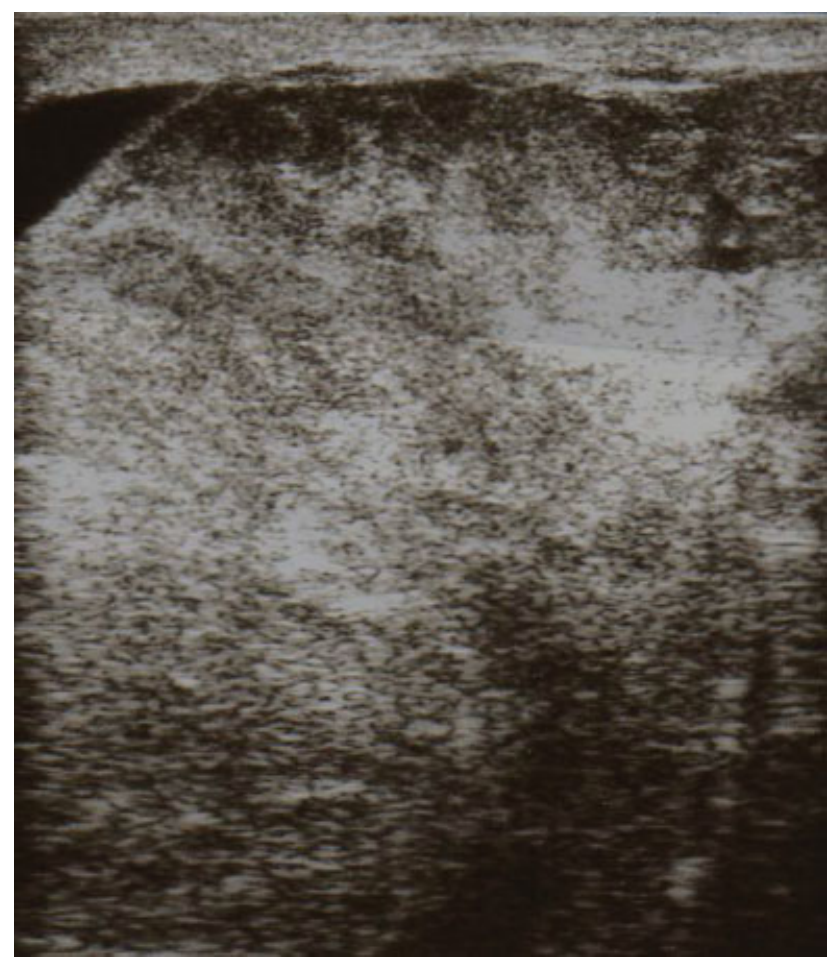

Fig. 2 Ecografía testicular: Teste izquierdo desestructurado, heterogéneo y de gran tamaño.
Posteriormente, se amplía estudio de imagen con tomografía computarizada (TC) toracoabdominal, caracterizando múltiples metástasis hepáticas, esplénicas y pulmonares. Se decide realizar punción-biopsia hepática ecodirigida de una de las LOE hepáticas (-Fig. 3), así como orquiectomía izquierda (-Fig. 4). Ambos resultados fueron compatibles con leiomiosarcoma de origen testicular mostrando células fusiformes con núcleo atípico. Se realizó inmunohistoquímica con positividad para marcadores musculares y desmina en ambas muestras (-Fig. 5).

Debido al estadio avanzado de la enfermedad, se remitió al paciente a Oncología para valoración de tratamiento quimioterápico adyuvante. Dadas las características del paciente se optó por un manejo sintomático.

El paciente falleció a los tres meses del diagnóstico.

\section{Discusión}

El leiomiosarcoma es una neoplasia maligna de origen mesenquimal, que se localiza en tejidos que contienen músculo liso. ${ }^{1}$ Supone aproximadamente un $7 \%$ de las neoplasias de tejidos blandos.

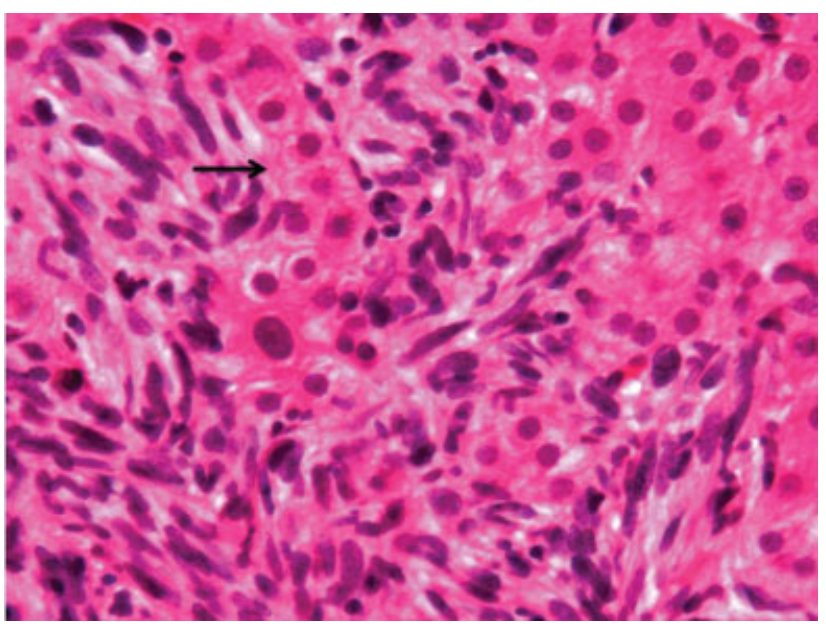

Fig. 3 Metástasis hepática de leiomiosarcoma: células fusiformes atípicas infiltrando entre las trabéculas de hepatocitos (flecha). H-E x 400. 


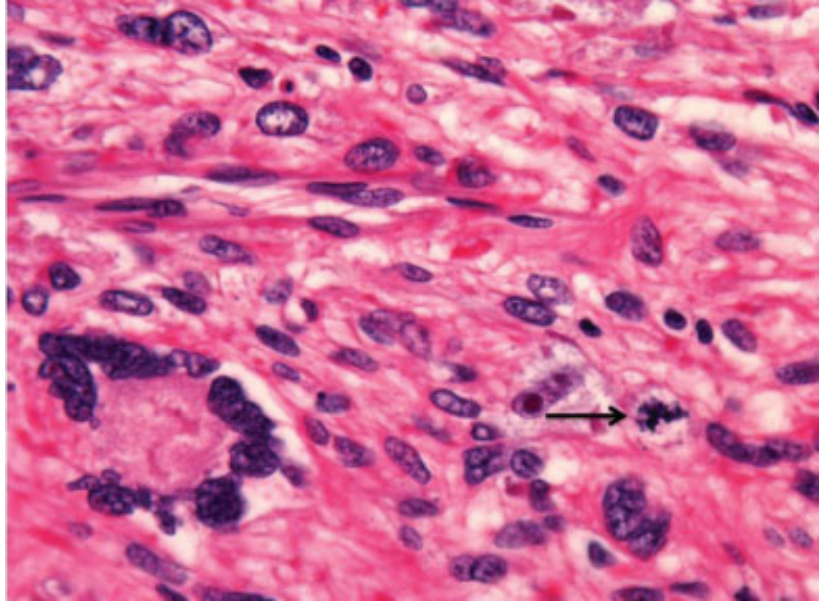

Fig. 4 Leiomiosarcoma testicular: células fusiformes de núcleos hipercromáticos, muy atípicos, a veces múltiples, con mitosis frecuente (flecha). H-E x 400.

Se identifica fundamentalmente en pacientes de mediana y avanzada edad, con predominio en útero y aparato digestivo, sobre todo con origen en grandes vasos $\mathrm{y}$ retroperitoneo.

Su localización en el aparato genitourinario masculino, concretamente en el testículo, se relaciona con antecedentes de radioterapia, inflamación crónica, ${ }^{8}$ uso de corticoides anabolizantes $^{9}$ o tumores de células germinales. La expresión clínica es similar a otras neoplasias testiculares.

El diagnóstico se establece en base a hallazgos histológicos e inmunohistoquímicos, que identifican células fusiformes del músculo liso maligno con núcleos atípicos positivos para la actina del músculo liso y desmina. ${ }^{10}$
Los subtipos histológicos incluyen leiomiosarcoma, rabdomiosarcoma, liposarcoma o sarcoma pleomórfico indiferenciado de alto grado.

Los tumores intraescrotales pueden ser testiculares (20\%) o paratesticulares (80\%), esos últimos con origen en el cordón espermático. Se ha recogido en la bibliografía, alrededor de un centenar de casos de localización paratesticular, habiéndose descrito la localización intratesticular en tan sólo una veintena de casos. ${ }^{5-10}$

La mayor parte de los casos de leiomiosarcoma testicular descritos fueron tumores localizados sin enfermedad a distancia, estadio I, cuyo tratamiento electivo fue la orquiectomía con una evolución favorable tras una prueba de imagen que garantizara la ausencia de enfermedad residual o a distancia. Incluso en esos casos tratados con orquiectomía, el pronóstico sería mejor que en el caso de tumores paratesticulares.

Debido al escaso número de casos de ese tipo de tumor diagnosticado, no existen recomendaciones específicas de tratamiento. Sin embargo, la literatura existente apoya la orquiectomía radical con posterior seguimiento estrecho mediante pruebas de imagen de forma semestral. ${ }^{2-5}$ Respecto a los casos de enfermedad avanzada, ${ }^{6,7}$ estadios II y III, la incertidumbre sobre el mejor tratamiento es mayor ya que la experiencia clínica es escasa. En esos casos, la opción con mejores resultados parece ser la orquiectomía combinada con quimioterapia adyuvante, ${ }^{7}$ ya que ese tipo de tumores es resistente a la radioterapia. ${ }^{10}$

En nuestro caso, el paciente no presentaba ninguno de los factores de riesgo relacionados con ese tumor testicular. En el momento del diagnóstico, se trataba de una enfermedad diseminada con múltiples metástasis, estadio III, por lo que sería subsidiario de asociar quimioterapia adyuvante. Sin embargo, dada la comorbilidad del paciente, se optó por un manejo conservador.
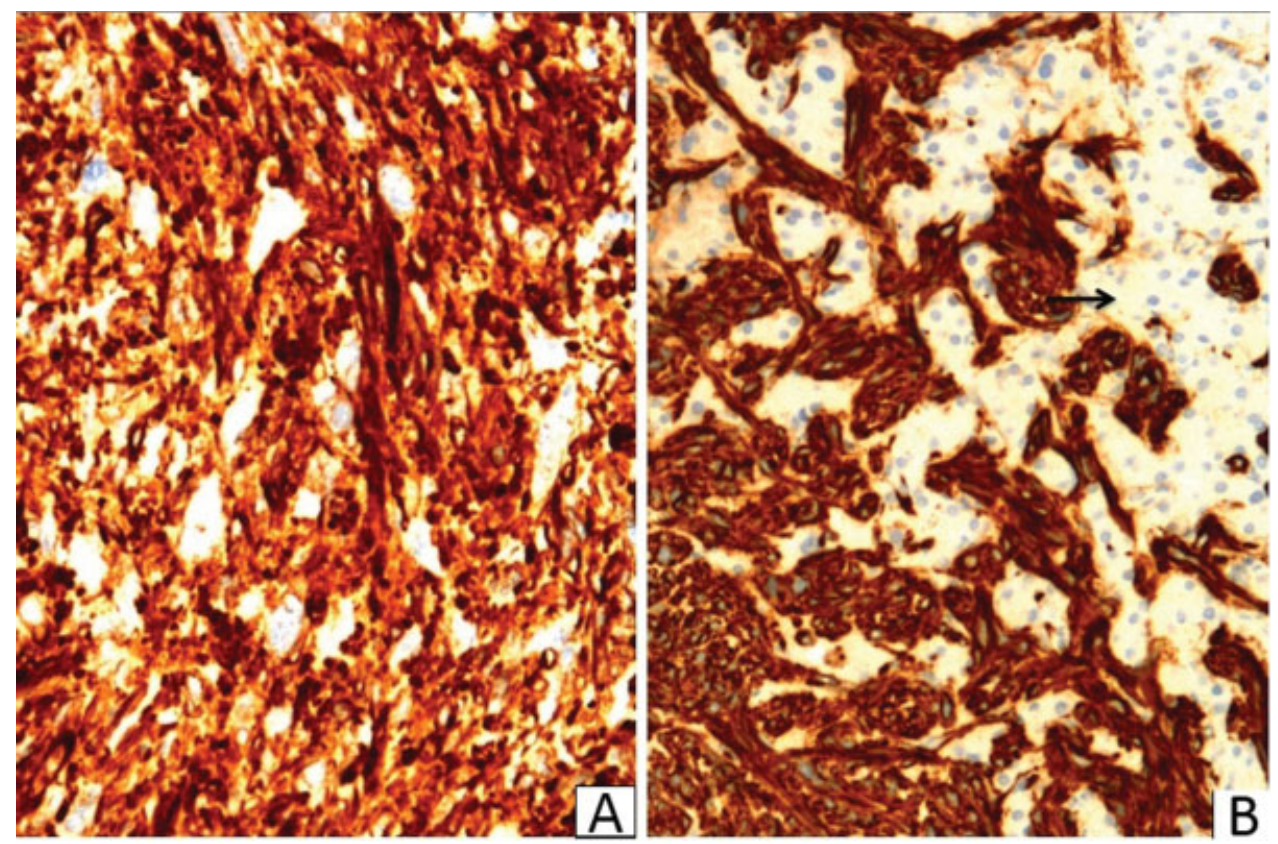

Fig. 5 Marcadores musculares inmunohistoquímicos intensamente positivos. (A) Leiomiosarcoma testicular. Desmina $\times 200$. (B) Metástasis hepática. HHF-35 $\times 200$. Véanse las trabéculas de hepatocitos negativas (flecha). 


\section{Conclusiones}

La punción hepática ecodirigida continúa siendo una herramienta de primera línea en el diagnóstico de la patología tumoral hepática metastásica, incluso en los casos de neoplasias raras como el leiomiosarcoma testicular.

Debido a la escasa experiencia actual no existen recomendaciones terapéuticas establecidas en este tumor, fundamentalmente en los estadios II y III, precisándose de más investigaciones en esa área.

Protección de Personas

Los autores declaran que para esta investigación, no se han realizado experimentos en seres humanos.

\section{Confidencialidad de los Datos}

Los autores declaran que han seguido los protocolos de su centro de trabajo sobre la publicación de datos de pacientes.

Derecho a la Privacidad y Consentimiento Informado Los autores declaran que en este artículo no aparecen datos de pacientes.

\section{Bibliografía}

1 DeVita VT, Lawrence TS, Rosenberg SA. Cancer: principles and practice of oncology. 9 ed. Part 8; Chapter 115; Lippincott Williams: London, 2012:1543

2 Takizawa A, Miura T, Fujinami K, Kawakami S, Osada Y, Kameda Y. Primary testicular leiomyosarcoma. Int J Urol 2005;12(06):596-598

3 Labanaris AP, Zugor V, Smiszek R, Nützel R, Kühn R. Primary leiomyosarcoma of the testis. A case report. Anticancer Res 2010; 30(05):1725-1726

4 Bostanci Y, Ozden E, Akdeniz E, et al. Primary testicular leiomyosarcoma. Can J Urol 2013;20(02):6730-6733

5 Abdullazade S, Kara O, Akdoğan B, Baydar DE. Primary low grade intratesticular leiomyosarcoma: case report and review of the literature. Turk Patoloji Derg 2013;29(03):227-230

6 Yoshimine S, Kono H, Nakagawa K, et al. Primary intratesticular leiomyosarcoma. Can Urol Assoc J 2009;3(06):E74-E76

7 Giridhar V, Kumar PB, Natarajan K, Hegde P. Testicular leiomyosarcoma with metastasis. Indian JUrol 2011;27(02):278-279

8 Ali Y, Kehinde EO, Makar R, Al-Awadi KA, Anim JT. Leiomyosarcoma complicating chronic inflammation of the testis. Med Princ Pract 2002;11(03):157-160

9 Froehner M, Fischer R, Leike S, Hakenberg OW, Noack B, Wirth MP. Intratesticular leiomyosarcoma in a young man after high dose doping with Oral-Turinabol: a case report. Cancer 1999;86(08):1571-1575

10 Hermans BP, Foster RS, Donohue JP. Paratesticular masses. AUA Update Ser 1998;17:290-295 